\title{
A multicenter phase I/II study of enzalutamide in Japanese patients with castration-resistant prostate cancer
}

\author{
Hideyuki Akaza ${ }^{1}$ Hirotsugu Uemura ${ }^{2}$ Taiji Tsukamoto $^{3} \cdot$ Seiichiro Ozono $^{4} \cdot$ \\ Osamu Ogawa ${ }^{5} \cdot$ Hideki Sakai $^{6} \cdot$ Mototsugu Oya $^{7} \cdot$ Mikio Namiki $^{8}$. \\ Satoshi Fukasawa ${ }^{9}$ - Akito Yamaguchi ${ }^{10}$ Hiroji Uemura ${ }^{11}$ ' Yasuo Ohashi ${ }^{12}$. \\ Hideki Maeda ${ }^{13} \cdot$ Atsushi Saito $^{13} \cdot$ Kentaro Takeda $^{13} \cdot$ Seiji Naito ${ }^{10}$
}

Received: 23 November 2015 / Accepted: 7 January 2016 / Published online: 21 January 2016

(c) The Author(s) 2016. This article is published with open access at Springerlink.com

\begin{abstract}
Background The safety, tolerability, pharmacokinetics (PK) and anti-tumor activity of enzalutamide were investigated in patients with castration-resistant prostate cancer (CRPC) in Japan through a multicenter phase I/II study.

Methods In phase I, patients with progressive metastatic CRPC received single, then multiple, ascending doses of enzalutamide 80, 160 or $240 \mathrm{mg} /$ day. After assessment of tolerability at multiple doses of $160 \mathrm{mg} / \mathrm{day}$ for 4 weeks, post-docetaxel patients with CRPC and measurable disease
\end{abstract}

Electronic supplementary material The online version of this article (doi:10.1007/s10147-016-0952-6) contains supplementary material, which is available to authorized users.

Hideyuki Akaza

akazah@med.rcast.u-tokyo.ac.jp

1 Strategic Investigation On Comprehensive Cancer Network, The University of Tokyo, 4-6-1 Komaba, Meguro-ku, Tokyo 153-8904, Japan

2 Department of Urology, Kinki University Faculty of Medicine, 377-2 Ono-Higashi, Osakasayama 589-8511, Japan

3 Department of Urology, Sapporo Medical University School of Medicine, S1 W17 Chuo-ku, Sapporo 060-8556, Japan

4 Department of Urology, Hamamatsu University School of Medicine, 1-20-1 Handayama, Higashi-ku, Hamamatsu 431-3192, Japan

5 Department of Urology, Kyoto University Graduate School of Medicine, Yoshida-Konoe-cho, Sakyo-ku, Kyoto 606-8501, Japan

6 Department of Urology, Nagasaki University Graduate School of Biomedical Sciences, 1-7-1 Sakamoto, Nagasaki 852-8501, Japan were enrolled into phase II; receiving long-term administration of enzalutamide $160 \mathrm{mg} /$ day.

Results Nine and 38 patients were enrolled in phase I and II, respectively. During phase I, enzalutamide was well tolerated in each cohort; PK parameters were similar to those of non-Japanese populations in other studies. By week 12, overall response rate was $5.3 \%$ and clinical benefit rate was $47.4 \%$. Prostate-specific antigen response rate $(\geq 50 \%$ reduction from baseline) was $28.9 \%$. Treatment-emergent adverse events reported in $>20 \%$ of patients in phase II were decreased weight, decreased appetite and constipation. No seizures were observed.

Conclusion Enzalutamide at $160 \mathrm{mg} /$ day was well tolerated, with PK and safety profiles similar to the

7 Department of Urology, Keio University School of Medicine, 35 Shinanomachi, Shinjuku-ku, Tokyo 160-8582, Japan

8 Department of Integrative Cancer Therapy and Urology, Kanazawa University Graduate School of Medical Sciences, 13-1 Takara-machi, Kanazawa 920-8641, Japan

9 Department of Urology, Chiba Cancer Center, 666-2 Nitona-Cho, Chuo-ku, Chiba 260-8717, Japan

10 Division of Urology, Harasanshin Hospital, 1-8 Taihakumachi, Hakata-ku, Fukuoka 812-0033, Japan

11 Department of Urology and Renal Transplantation, Yokohama City University Medical Center, 4-57 Urafune, Minami-ku, Yokohama 232-0024, Japan

12 Department of Integrated Science and Engineering for Sustainable Society, Chuo University, 1-13-27 Kasuga, Bunkyo-ku, Tokyo 112-8551, Japan

13 Astellas Pharma Inc, 2-5-1 Nihonbashi-Honcho, Chuo-Ku, Tokyo 103-8411, Japan 
non-Japanese population. Anti-tumor activity was observed in post-docetaxel Japanese patients with metastatic CRPC. Apparent differences in anti-tumor activity compared with the AFFIRM study (a phase III trial in a diverse population of patients with CRPC post-docetaxel) may be attributed to differences in treatment history prior to starting enzalutamide. Particularly in Japan, the influence of sequence in hormone treatments, including combined androgen blockade therapy, should be considered.

Trial registration: ClinicalTrials.gov NCT01284920.

Keywords Androgen receptor inhibitor - Enzalutamide . Metastatic castration-resistant prostate cancer

\section{Introduction}

As prostate cancer growth is dependent on androgens, androgen deprivation therapy (ADT), which includes surgical castration or medical therapy with gonadotropinreleasing hormone $(\mathrm{GnRH})$ agonists or $\mathrm{GnRH}$ antagonists, is standard therapy for patients with metastatic prostate cancer recurrence after definitive therapy, or inoperable prostate cancer. In Japan, it is common practice in primary ADT to use androgen blockade combined with bicalutamide, a non-steroidal anti-androgen [1-4]. Progression of the disease despite castrate levels of testosterone under primary ADT is considered castrationresistant prostate cancer (CRPC) [5] and it generally represents a transition to the lethal state of the disease. CRPC is frequently treated with hormone therapy alternating with anti-androgens, low dose steroids or estrogenic compounds [6]. However, prolonged survival of patients with CRPC by these secondary hormonal treatments is not confirmed [7]. Until early 2014, docetaxel plus prednisone were the only approved drugs for patients with advanced CRPC in Japan [8, 9].

Recent treatment options that have demonstrated a survival improvement in patients with metastatic CRPC include cabazitaxel plus prednisone [10] and abiraterone plus prednisone [11, 12]. Enzalutamide [13, 14], sipuleucel-T [15] and radium Ra 223 dichloride [16] have also been approved for use in several countries.

Enzalutamide is a novel androgen receptor inhibitor that significantly prolongs survival of men with CRPC regardless of prior docetaxel therapy [13]. Enzalutamide inhibits multiple steps in the androgen receptor signaling pathway and is devoid of agonist activity in preclinical models [17]. Preclinical pharmacology studies have demonstrated that enzalutamide competitively inhibits androgen-induced receptor activation, nuclear translocation of activated androgen receptors, and the association of the activated androgen receptor with chromatin, even in the setting of androgen receptor over-expression and in prostate cancer cells resistant to anti-androgens [17].

The efficacy of enzalutamide was evaluated in two multinational phase III studies in men with metastatic CRPC; AFFIRM and PREVAIL. The AFFIRM trial showed overall survival (OS) benefit of enzalutamide in post-docetaxel patients with metastatic CRPC versus placebo. Median survival was 18.4 months with enzalutamide and 13.6 months with placebo [hazard ratio $0.63 ; 95 \%$ confidence interval (CI) $0.53-0.75 ; p<0.001]$ [14]. The PREVAIL trial confirmed clinical benefit of enzalutamide in chemotherapynaïve patients with metastatic CRPC. The hazard ratio of OS and radiographic progression-free survival (rPFS) were $0.71(95 \%$ CI $0.60-0.84 ; p<0.001)$ and $0.19(95 \%$ CI $0.15-0.23 ; p<0.001)$, respectively [13]. Median OS was 32.4 months with enzalutamide and 30.2 months with placebo. Median rPFS was not reached with enzalutamide and was 3.9 months with placebo [13].

The present phase I/II clinical study evaluated the safety, tolerability and pharmacokinetics (PK) of enzalutamide in patients with CRPC and the anti-tumor activity and safety of enzalutamide in Japanese post-docetaxel patients with CRPC to provide supporting data for the regulatory approval of enzalutamide in Japan.

\section{Patients and methods}

\section{Study design}

This was a multicenter, open-label, uncontrolled study of orally administered enzalutamide, involving two phases (http://ClinicalTrials.gov NCT01284920). Phase I involved dose escalation in patients with CRPC. Phase II involved dose expansion in post-docetaxel patients with CRPC.

All participating sites obtained approval for conducting the study by their institutional review boards. The study was conducted in accordance with the Declaration of Helsinki, Good Clinical Practice Guidelines and the Pharmaceutical Affairs Law in Japan. All patients provided written informed consent to participate in the study.

In phase I, patients received a single dose of enzalutamide (80, 160 or $240 \mathrm{mg} /$ day) and blood samples for PK analysis were collected over 7 days. Subsequently, patients received multiple doses of enzalutamide at the same dosage levels as in the single-dose period. Tolerability was evaluated 29 days after initiation of repeat dosing by an independent data monitoring committee. Patients who received $240 \mathrm{mg}$ in the single-dose period subsequently received multiple doses of $160 \mathrm{mg} /$ day (the recommended dose in the AFFIRM study [12]). Following the evaluation of enzalutamide tolerability and PK parameters after single and multiple doses at $160 \mathrm{mg}$ in phase I, an open-label, 
uncontrolled phase II study was initiated to evaluate the efficacy, safety and PK in patients receiving enzalutamide $160 \mathrm{mg} /$ day. The study design was discussed with the Pharmaceuticals and Medicine Devices Agency from a perspective of regulatory approval of enzalutamide in Japan. Consequently, overall response rate was selected to be the primary outcome variable in this study, thereby requiring enrolment of patients with measureable disease into the study.

\section{Patients}

Patients with metastatic CRPC who had disease progression while on castration therapy were eligible for participation. Patients had to have received ADT with a GnRH analogue or a bilateral orchiectomy with serum testosterone level maintained within castration level $(\leq 50 \mathrm{ng} / \mathrm{dL})$.

The criteria used to define disease progression for trial entry are available in the Online Resource. Eligible patients had Eastern Cooperative Oncology Group performance status of 0 or 1 (or 2 if only due to metastatic bone pain at the screening). Post-chemotherapy patients had to have received prior chemotherapy with docetaxel and no more than two prior chemotherapy regimens. In particular for phase II, patients had to have measurable lesions as determined by response evaluation criteria for solid tumors (RECIST) guidelines.

Exclusion criteria were history of seizure (including any febrile seizure, loss of consciousness or transient ischemic attack within 12 months prior to initiation of study drug) or any condition that may predispose to seizure. The complete list of exclusion criteria is available in the Online Resource.

\section{Assessments}

The primary outcome in anti-tumor activity in phase II was best overall response by 12 weeks; defined by RECIST guidelines as complete response (CR) or partial response (PR) and assessed by an investigator. Confirmation of CR or PR was required by a subsequent scan at least 4 weeks later. When the investigator confirmed CR or PR, the assessment was finally evaluated by an independent RECIST assessment committee. Measurements had to meet the stable disease criteria by day 85 for determination of stable disease. Radiographic imaging for the target region was conducted at the screening visit, on day 29, day 57 and day 85 , and at each subsequent visit every 84 days. Bone scans were examined at the screening visit and at each 84-day visit.

The secondary endpoint was prostate-specific antigen (PSA) response rate (proportion of subjects with $\geq 50 \%$ decline in serum PSA from baseline). Serum PSA measurements were conducted at the screening visit and at each subsequent visit every 28 days.

Safety was evaluated from the start of study treatment to 30 days after completion of the study treatment. All adverse events (AEs) were recorded using the National Cancer Institute Common Terminology Criteria for Adverse Events (NCI CTCAE), version 4.0, and Medical Dictionary for Regulatory Activities (MedDRA), version 14.1. Laboratory values, vital signs, body weight and 12-lead echocardiograms were assessed at predefined time points.

Blood samples were collected at predefined time points in phase I and phase II. Plasma concentrations of enzalutamide and its active metabolite, $N$-desmethyl enzalutamide, were determined by a validated bioanalytical method based on liquid chromatography combined with mass spectrometry [18]. PK parameters were estimated by non-compartmental methods in WinNonlin ${ }^{\circledR}$ (Pharsight Corp., Palo Alto, CA, USA) and included maximum plasma concentration $\left(\mathrm{C}_{\max }\right)$ and area under the plasma concentration-time curve from time 0 to infinity $\left(\mathrm{AUC}_{\infty}\right)$. To investigate potential PK differences between Japanese and non-Japanese patients, the PK data were compared with PK data from AFFIRM.

\section{Statistical analysis}

The number and percentage of patients with a best overall response by day 85 and two-sided 90 and $95 \%$ ClopperPearson CIs were used in the primary analysis. A waterfall plot of maximum percent change from baseline of serum PSA was created. The number and percentage of patients with best PSA response at time of nadir were summarized. All data processing, summarization and analyses were performed using SAS Drug Development, version 3.4, and PCSAS, version 9.1.3. All analyses were performed by the sponsor using data obtained by the cut-off date of 12 July 2012 .

\section{Post hoc analysis}

An additional post hoc exploratory analysis was conducted to further compare enzalutamide anti-tumor activity in Japanese patients with non-Japanese patients with measurable disease from the AFFIRM study. The best overall response by number of prior hormonal therapy lines, and defined by RECIST and PSA response rate, was calculated.

\section{Results}

\section{Patients}

In phase I, three patients were assigned to each of the 80 , 160 and $240 \mathrm{mg}$ groups. The median duration of exposure in each group was 584.0, 171.0 and 252.0 days, respectively. 
Table 1 Summary of demographics and other baseline characteristics

Demographic/characteristic $\quad$ Phase I $(N=9) \quad$ Phase II $(N=38)$

\begin{tabular}{lll}
\hline Demographic/characteristic & Phase $\mathrm{I}(N=9)$ & Phase $\mathrm{II}(N=38)$ \\
\hline Age (years) & & \\
Median & 73.0 & 71.5 \\
Min-max & $62-86$ & $50-85$
\end{tabular}

Height $(\mathrm{cm})$

$\begin{array}{lll}\text { Median } & 166.0 & 165.7 \\ \text { Min-max } & 156.2-174.4 & 153.4-181.0\end{array}$

Weight $(\mathrm{kg})$

\section{Median}

71.2

Min-max

$49.2-88.9$

ECOG PS ${ }^{\mathrm{a}}$

Grade 0

8 (88.9)

$1(11.1)$

Grade 1

Total Gleason score ${ }^{\mathrm{b}}$ at initial diagnosis ${ }^{\mathrm{a}}$

Low, 2-4

Medium, 5-7

High, 8-10

Unknown

Clinical tumor stage $(\mathrm{T})^{\mathrm{c}}$ at initial diagnosis ${ }^{\mathrm{a}}$

TX
T0
T1
T2
T3
T4
Unknown
Clinical lymph node stage at initic

at initial diagnosis ${ }^{\mathrm{a}}$

$\begin{array}{ll}\text { NX } & 2(22.2) \\ \text { N0 } & 3(33.3) \\ \text { N1 } & 4(44.4) \\ \text { Unknown } & 0\end{array}$

Distant metastasis $(\mathrm{M})^{\mathrm{c}}$ at initial diagnosis ${ }^{\mathrm{b}}$

\begin{tabular}{lll} 
MX & 0 & $1(2.6)$ \\
M0 & $3(33.3)$ & $17(44.7)$ \\
M1 & $6(66.7)$ & $19(50.0)$ \\
Unknown & 0 & $1(2.6)$ \\
Number of bone metastases & & \\
0 & $3(33.3)$ & $8(21.1)$ \\
1 & $1(11.1)$ & $1(2.6)$ \\
$2-4$ & $1(11.1)$ & $6(15.8)$ \\
$5-9$ & 0 & $7(18.4)$ \\
$\geq 10$ & $4(44.4)$ & $16(42.1)$ \\
Anti-androgen withdrawal syndrome & \\
Yes & 0 & $4(10.5)$ \\
Stage of prostate cancer ${ }^{\mathrm{a} b}$ & & \\
Localized & $1(11.1)$ & $6(15.8)$ \\
Locally advanced & $2(22.2)$ & $11(28.9)$ \\
Metastatic & $6(66.7)$ & $19(50.0)$ \\
Not classifiable & 0 & $2(5.3)$ \\
\hline
\end{tabular}

Table 1 continued

\begin{tabular}{lll}
\hline Demographic/characteristic & Phase I $(N=9)$ & Phase II $(N=38)$ \\
\hline PSA at baseline (ng/mL) & & \\
Mean (SD) & $634.82(1403.52)$ & $174.94(307.97)$ \\
Median & 21.60 & 65.80 \\
Duration of disease at screening (months) & \\
Mean (SD) & $47.36(22.23)$ & $63.11(38.15)$ \\
Median & 39.93 & 52.83 \\
\hline
\end{tabular}

ECOG PS Eastern Cooperative Oncology Group performance status, $P S A$ prostate-specific antigen, $S D$ standard deviation

${ }^{\text {a }}$ Number $(\%)$ of patients

b Gleason [27]

c Classified using the TNM classification [28] as follows: localized, $\mathrm{T} 1 / 2$ and (NX or N0) and M0; locally advanced, T3/4 and (NX or N0) and M0 or N1 and M0; metastatic, M1; Not classifiable, others

Thirty-eight post-docetaxel patients with CRPC and measurable disease as defined by RECIST were enrolled into phase II at a dose of $160 \mathrm{mg} /$ day. Median duration of exposure was 121 days. No remarkable differences were observed in the demographic and clinical baseline characteristics between the phase I and phase II study populations (Table 1). Patients in phase II were heavily pretreated, with $>90 \%$ having had $\geq 4$ prior hormonal treatments (Table 2). Eight of 38 patients $(21.1 \%)$ in phase II discontinued due to AEs. Of these, five patients withdrew due to disease progression (Table 3). All

Table 2 Prior treatments for prostate cancer in phase II

\begin{tabular}{lll}
\hline Parameter & Category/statistic & Phase II $(N=38)$ \\
\hline $\begin{array}{l}\text { Cancer treatment history, } \\
\text { radiation }\end{array}$ & Yes & $19(50.0 \%)$ \\
$\begin{array}{l}\text { Cancer treatment history, } \\
\text { procedure }\end{array}$ & Yes & $6(15.8 \%)$ \\
$\begin{array}{l}\text { Quantity of prior hormone } \\
\text { therapy lines }\end{array}$ & 3 & $3(7.9 \%)$ \\
& 4 & $5(13.2 \%)$ \\
& 5 & $13(34.2 \%)$ \\
& 6 & $11(28.9 \%)$ \\
$\begin{array}{l}\text { Typical prior hormone therapy } \\
\text { other than GnRH analogue }\end{array}$ & Bicalutamide & $6(15.8 \%)$ \\
& Flutamide & $29(100.0 \%)$ \\
& Estramustine & $30(78.9 \%)$ \\
Number of prior chemotherapy & 1 & $38(100 \%)$ \\
regimens & Docetaxel & $8(21.1 \%)$ \\
Duration of prior docetaxel & 2 & $30(78.9 \%)$ \\
(days) & Median & 198 \\
\hline
\end{tabular}

GnRH gonadotropin-releasing hormone

a Sum of prior hormonal treatment agents including castration therapy 
Table 3 Primary reasons for discontinuation

\begin{tabular}{lcc}
\hline Category and reason & Phase I $(N=9)$ & Phase II $(N=38)$ \\
\hline Discontinuation in multiple-dose period (early termination), $n(\%)$ \\
Adverse event & 0 & $5(13.2)$ \\
Worsening of disease & $1(11.1)$ & $5(13.2)$ \\
Withdrawal by subject & 0 & $2(5.3)$ \\
Discontinuation in overall study, $n(\%)$ & \\
Adverse event & 0 & $8(21.1)$ \\
Worsening of disease & $5(55.6)$ & $18(47.4)$ \\
Withdrawal by subject & 0 & $2(5.3)$ \\
\hline
\end{tabular}

Table 4 Best overall responses by day 85

\begin{tabular}{ll}
\hline Best overall response & $\begin{array}{l}\text { Evaluation by RECIST assess- } \\
\text { ment committee and investigator } \\
(N=38)\end{array}$ \\
\hline $\mathrm{CR}, n$ & 0 \\
$\mathrm{PR}, n(\%)$ & $2(5.3)$ \\
Stable disease, $n(\%)$ & $16(42.1)$ \\
$\mathrm{PD}, n(\%)$ & $16(42.1)$ \\
Not evaluated & $4(10.5)$ \\
$\mathrm{CR}$ or PR, $n(\%)$ (response rate) & $2(5.3)$ \\
$95 \% \mathrm{CI}^{\mathrm{b}}$ & $0.6-17.7 \%$ \\
$90 \% \mathrm{CI}^{\mathrm{b}}$ & $0.9-15.7 \%$ \\
$\mathrm{CR}$ or PR or stable disease, $n(\%)$ & $18(47.4)$ \\
$($ disease control rate) & \\
$95 \% \mathrm{CI}^{\mathrm{b}}$ & $31.0-64.2 \%$ \\
$90 \% \mathrm{CI}^{\mathrm{b}}$ & $33.3-61.8 \%$ \\
\hline
\end{tabular}

Tumor response (overall response) for each patient was assessed by the investigator and subsequently evaluated by an independent RECIST assessment committee (when the investigator assessed that a patient had been accomplished CR or PR)

$C R$ complete response, $P D$ progressive disease, $P R$ partial response

${ }^{a}$ When there were evaluation data from both the RECIST committee and investigator, RECIST assessment committee data were adopted

b Based on exact binomial confidence interval (Clopper-Pearson) patients had received complete androgen blockade $(\mathrm{CAB})$ therapy with bicalutamide soon after their initial diagnosis of prostate cancer. Overall, $42.1 \%$ had $>10$ bone metastases and all patients had measurable disease by RECIST (Table 1).

\section{Anti-tumor activity}

\section{Response}

The best overall response rate (CR and PR) by day 85 , as evaluated by the RECIST assessment committee and investigators, was seen in $5.3 \%$ of patients (two out of 38; $95 \%$ CI $0.6-17.7 \%$; $90 \%$ CI $0.9-15.7 \%$ ). The best overall disease control rate (CR plus PR plus stable disease) by day 85 was $47.4 \%$ of patients (18 out of 38 ; $95 \%$ CI 31.0 $64.2 \%$; $90 \%$ CI 33.3-61.8\%) (Table 4). The rate with which the sum of diameters of target lesions was reduced by $\geq 30 \%$ was $18.4 \%$ ( 7 out of 38 patients).

PSA

Eleven out of 38 patients in phase II $(28.9 \%$; $95 \%$ CI $15.4-45.9 \%$ ) had a $\geq 50 \%$ decrease in PSA levels at the time of nadir, as compared with baseline (Fig. 1; Table 5).

\section{Safety}

The most frequent treatment-emergent AEs (TEAEs) with an incidence of $\geq 20 \%$ across both phases were weight decrease $(36.2 \%)$, decreased appetite $(27.7 \%)$ and constipation $(25.5 \%)$ (Table 6). Of the adverse drug reactions reported in $\geq 10 \%$ of patients, those considered to be related to the study drug were hypertension $(14.9 \%)$, constipation (14.9\%), fatigue (12.8\%), decreased appetite $(12.8 \%)$, weight decrease $(10.6 \%)$ and electrocardiogram QT prolonged (10.6\%). None
Fig. 1 Waterfall plot of maximum percent change from baseline of serum PSA in phase II. $P S A$, prostate-specific antigen

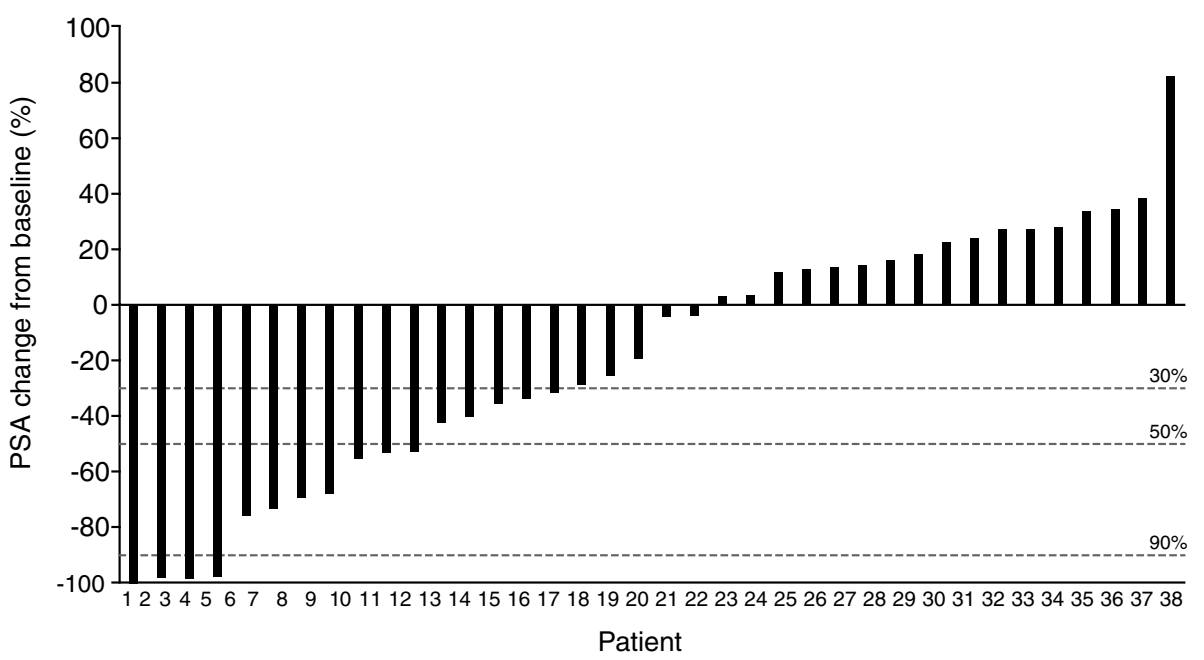


of the TEAEs resulted in death and no seizures were reported. The most common serious TEAE was cancer pain $(N=3)$ (Table 7$)$.

Table 5 Best PSA response at time of nadir

\begin{tabular}{ll}
\hline Response & $160 \mathrm{mg} / \mathrm{day}(N=38)$ \\
\hline Decline from baseline, patients, $n(\%)$ & \\
$\geq 30 \%$ & $15(39.5)$ \\
$95 \% \mathrm{CI}$ & $24.0-56.6 \%$ \\
$\geq 50 \%$ & $11(28.9)$ \\
$95 \% \mathrm{CI}$ & $15.4-45.9 \%$ \\
$\geq 90 \%$ & $4(10.5)$ \\
$95 \% \mathrm{CI}$ & $2.9-24.8 \%$ \\
\hline
\end{tabular}

\section{Pharmacokinetics}

Enzalutamide was absorbed rapidly after oral administration in Japanese patients and the PK was dose-proportional after a single dose ranging from 80 to $240 \mathrm{mg}$ (Fig. 2). The PK profile of a single dose of enzalutamide in Japanese patients was similar to that of non-Japanese patient data from the first enzalutamide study in humans (phase I/ II study; http://ClinicalTrials.gov NCT00510718) (Fig. 2) [19]. PK profiles of the sum of enzalutamide plus its active metabolite in plasma were similar between Japanese patients from the current study and non-Japanese populations from AFFIRM (Fig. 3).

$C I$ confidence interval, PSA prostate-specific antigen

Table 6 Common adverse events (reported in at least $10 \%$ of patients in total)

\begin{tabular}{|c|c|c|c|c|c|c|}
\hline \multirow[t]{2}{*}{$\begin{array}{l}\text { MedDRA, version 14.1, } \\
\text { preferred term }\end{array}$} & \multicolumn{3}{|l|}{ All adverse events } & \multicolumn{3}{|c|}{$\begin{array}{l}\text { Adverse events considered to be related to } \\
\text { study drug }\end{array}$} \\
\hline & Phase I $(N=9)$ & Phase II $(N=38)$ & Total $(N=47)$ & Phase I $(N=9)$ & Phase II $(N=38)$ & Total $(N=47)$ \\
\hline Overall & $9(100.0)$ & 36 (94.7) & 45 (95.7) & 7 (77.8) & $24(63.2)$ & $31(66.0)$ \\
\hline Weight decreased & $1(11.1)$ & $16(42.1)$ & $17(36.2)$ & 0 & $5(13.2)$ & $5(10.6)$ \\
\hline Decreased appetite & $3(33.3)$ & $10(26.3)$ & $13(27.7)$ & $2(22.2)$ & $4(10.5)$ & $6(12.8)$ \\
\hline Constipation & $2(22.2)$ & $10(26.3)$ & $12(25.5)$ & $1(11.1)$ & $6(15.8)$ & $7(14.9)$ \\
\hline Hypertension & $3(33.3)$ & $6(15.8)$ & $9(19.1)$ & $3(33.3)$ & $4(10.5)$ & $7(14.9)$ \\
\hline Cancer pain & $1(11.1)$ & $8(21.1)$ & $9(19.1)$ & 0 & $1(2.6)$ & $1(2.1)$ \\
\hline Nausea & $4(44.4)$ & $5(13.2)$ & $9(19.1)$ & $1(11.1)$ & $2(5.3)$ & $3(6.4)$ \\
\hline Electrocardiogram QT prolonged & 0 & $6(15.8)$ & $6(12.8)$ & 0 & $5(13.2)$ & $5(10.6)$ \\
\hline Fatigue & $2(22.2)$ & $4(10.5)$ & $6(12.8)$ & $2(22.2)$ & $4(10.5)$ & $6(12.8)$ \\
\hline Nasopharyngitis & 1 (11.1) & $5(13.2)$ & $6(12.8)$ & 0 & 0 & 0 \\
\hline Pyrexia & $1(11.1)$ & $4(10.5)$ & $5(10.6)$ & $1(11.1)$ & 0 & $1(2.1)$ \\
\hline Somnolence & 0 & $5(13.2)$ & $5(10.6)$ & 0 & $1(2.6)$ & $1(2.1)$ \\
\hline Rash & 0 & $5(13.2)$ & 5 (10.6) & 0 & $1(2.6)$ & $1(2.1)$ \\
\hline
\end{tabular}

Number of patients (\%)

MedDRA Medical Dictionary for Regulatory Activities

Table 7 Serious treatmentemergent adverse events (with an incidence of $\geq 2$ events in the study)

\begin{tabular}{llc}
\hline MedDRA, version 14.1, preferred term & Phase I total $^{\mathrm{a}}(N=9)$ & Phase II $160 \mathrm{mg}(N=38)$ \\
\hline Overall & $2(22.2)$ & $13(34.2)$ \\
Cancer pain & $1(11.1)$ & $2(5.3)$ \\
Anemia & 0 & $2(5.3)$ \\
Disseminated intravascular coagulation & 0 & $2(5.3)$ \\
General physical health deterioration & 0 & $2(5.3)$ \\
Cellulitis & 0 & $2(5.3)$ \\
Tumor pain & 0 & $2(5.3)$ \\
Bladder tamponade & 0 & $2(5.3)$ \\
\hline
\end{tabular}

Number of patients (\%)

MedDRA Medical Dictionary for Regulatory Activities

${ }^{a}$ In phase I, safety data from single doses $(80,160$ and $240 \mathrm{mg}$ ) and multiple doses (80 and $160 \mathrm{mg}$ ) are included. All patients in the $240 \mathrm{mg}$ group received enzalutamide at a dose of $160 \mathrm{mg}$ after single dosing 

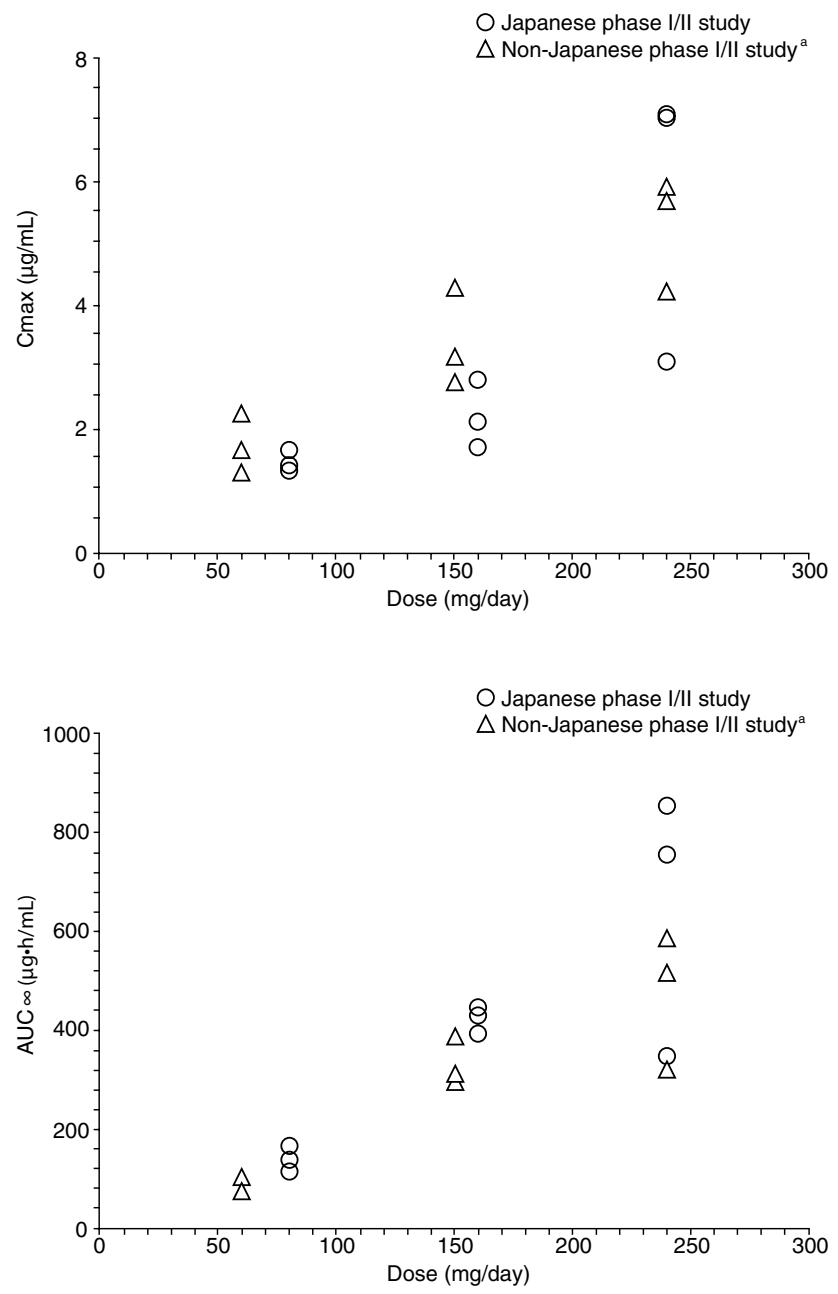

Fig. 2 Comparison of individual enzalutamide $C_{\max }$ and $A U C_{\infty}$ during single-dosing. ${ }^{\text {a }}$ Phase I, open-label, dose-escalation safety and pharmacokinetic study of enzalutamide in patients with CRPC conducted overseas [19]

\section{Post hoc analysis}

An exploratory post hoc analysis compared the subgroup of non-Japanese patients with measurable disease from AFFIRM $[N=446$ out of 800 enzalutamide-treated patients (cut-off date: 25 September 2011)] and Japanese patients from the current study $(N=38)$. The quantity of prior hormonal therapy lines used as prostate cancer treatment in the two studies, which excluded medical or surgical castration therapy, is available in the Online Resource in Table S1. While approximately $90 \%$ of patients in the AFFIRM study had received $\leq 2$ hormonal therapy lines, approximately $90 \%$ of patients in the current study had received $\geq 3$ hormonal therapy lines. Best overall response rate, by RECIST, and PSA response rate for each amount of prior hormonal therapy lines are available in the Online Resource in Table S2 and Table S3, respectively.

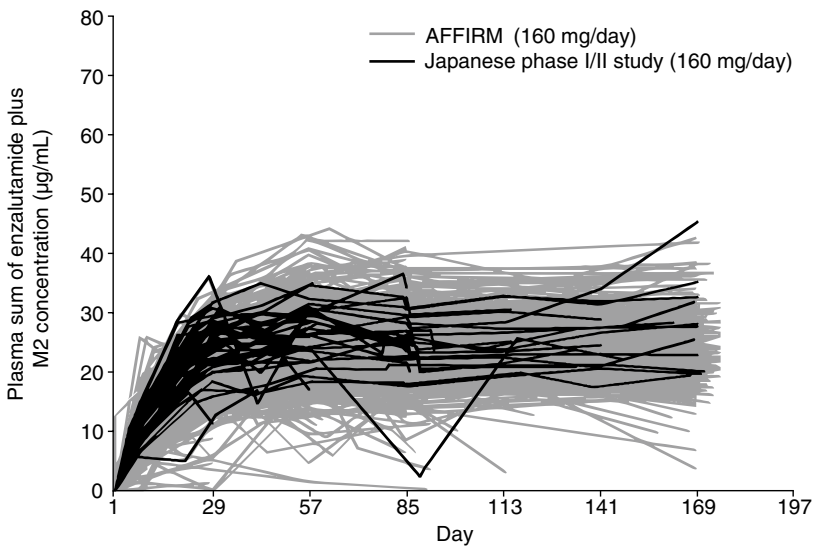

Fig. 3 Individual trough plasma sum of enzalutamide and active metabolite concentration versus time plot in the Japanese phase I/II and AFFIRM (International, phase III, randomized, double-blind, placebo-controlled study of enzalutamide in patients with prostate cancer who had previously been treated with one or two chemotherapy regimens, at least one of which contained docetaxel [14]) studies, up to day 169

\section{Discussion}

In patients who receive primary androgen deprivation therapy, the proportion of patients with high risk and/ or advanced prostate cancer is higher in Japan than in the United States [2]. A randomized, controlled study of primary ADT by $\mathrm{CAB}$ with chemical castration by $\mathrm{GnRH}$ agonist and bicalutamide $80 \mathrm{mg}$ in Japanese patients showed significant prolongation of OS compared with chemical castration alone [20]. Based on this result, CAB is used in Japan as a standard initial therapy for high-risk or progressive prostate cancer $[2,4]$.

Results from this first clinical study of enzalutamide in the Japanese post-docetaxel CRPC patient population showed that enzalutamide was well tolerated when orally administered at a dose of $160 \mathrm{mg}$ once daily. PK of enzalutamide was dose-proportional in the doses ranging from 80 to $240 \mathrm{mg}$ and similar to PK data from non-Japanese patients. Furthermore, enzalutamide administered orally at $160 \mathrm{mg}$ once daily had anti-tumor activity in Japanese postchemotherapy patients with CRPC, in terms of best overall response or tumor-shrinking tendency and PSA response.

However, this study did not achieve radiographic and PSA response rates as high as those in AFFIRM. The radiographic response rate by day 85 was $5.3 \%$ in the current study versus $28.9 \%$ in AFFIRM. The PSA response rate ( $\geq 50 \%$ reduction from baseline) was $54.0 \%$ in AFFIRM, compared with $28.9 \%$ in this study. Differences between the two studies (i.e., patient populations enrolled, study setting and design, patient samples in each trial) may account for the lower radiographic and PSA response rates. As prior docetaxel exposure received by patients in 
this study (median, 198 days; approximately 9-10 cycles) was similar to that reported by patients in the AFFIRM trial (median, 8.5 cycles) [14], the most important difference could be that patients in the current study had received more hormonal therapy lines prior to enzalutamide compared with those in AFFIRM. With the exclusion of castration therapies, approximately $90 \%$ of patients in this study had received $\geq 3$ prior hormonal therapy lines (i.e., CAB, anti-androgen alternative therapy, steroids, and estrogens), whereas patients typically received $\leq 2$ lines in AFFIRM. This difference may be related to the recommended treatment strategy in Japan, which includes extensive exposure to $\mathrm{CAB}$, with bicalutamide $80 \mathrm{mg}$ as the primary ADT and further treatment with alternating hormone therapy. This observation is also supported by the results of a previous surveillance study by the Japan Study Group of Prostate Cancer (J-CaP) that considered the current status of endocrine therapy for prostate cancer [4]. Of the 3337 patients who initially received primary ADT, 2477 patients $(74.2 \%)$ were given $\mathrm{CAB}$ in the J-Cap surveillance [4]. The pattern of primary ADT usage was more common in Japan than in the United States and primary ADT by CAB was associated with better survival than other forms of primary ADT in Japanese high-risk patients [2]. Although extensive direct comparisons between Japan and the United States are not possible, there are some differences between the two countries in the initial prostate cancer treatment selection and outcome $[2,4]$.

Furthermore, although hormonal treatments have been the mainstay of treatment in advanced prostate cancer, recent data suggest potential development of cross-resistance after multiple lines of hormonal therapy [21, 22]. In the first-in-man enzalutamide phase I/II study in patients in the United States, the rate of PSA decline of $\geq 50 \%$ was significantly lower in patients with previous ketoconazole treatment versus those without [37\% (95\% CI 25-50\%) versus $71 \%$ (95\% CI 60-81\%; $p=0.0007)$ ] [19]. Moreover, a study of abiraterone acetate plus prednisone showed that patients with prior exposure to ketoconazole had a lower percentage of PSA decline of $\geq 50 \%$ compared with ketoconazole-naïve patients [23]. In the same study, time to PSA progression was shorter in patients with prior ketoconazole exposure compared with ketoconazole-naïve patients [23]. In addition, there have been several recent reports on cross-tolerance between abiraterone plus prednisone and enzalutamide. Reports from compassionate use programs involving heavily pretreated patients with metastatic CRPC suggest reduced efficacy for both enzalutamide and abiraterone in comparison to the efficacy reported in clinical trials $[24,25]$. Furthermore, results of a recent study in 32 patients suggested a potential effect of androgen receptor splice variant-7 on primary treatment resistance, observed with abiraterone plus prednisone or enzalutamide [26].
Enzalutamide showed good tolerability in Japanese patients, with PK and safety profiles similar to those in nonJapanese populations included in other enzalutamide studies. The differences in anti-tumor activity observed in this study versus the AFFIRM trial may be attributed to differences in the study design and patients' backgrounds in each trial. In particular, they may be attributed to differences in treatment history prior to starting enzalutamide. This may require further investigation to define the optimal timing and treatment strategy of enzalutamide for patients with CRPC. Particularly in Japan, the influence of sequence for hormone treatments, including CAB therapy, should be considered.

Acknowledgments We thank the patients in the study for their cooperation, along with the following investigators: Hiroshi Kitamura, Sapporo Medical University Hospital; Hideo Saito, Tohoku University Hospital; Senji Hoshi, Yamagata Prefectural Central Hospital; Kazuhiro Suzuki, Gunma University Hospital; Takeshi Ueda, Chiba Cancer Center; Tomohiko Ichikawa, Chiba University Hospital; Yataro Yamanaka, Nihon University Itabashi Hospital; Takashi Fukagai, Showa University Hospital; Hirohiko Nagata, Keio University Hospital; Hiroyuki Fujimoto, National Cancer Center Hospital; Shin Egawa, Jikei University Hospital; Takatsugu Okegawa, Kyorin University Hospital; Hiroji Uemura, Yokohama City University Hospital; Tsutomu Nishiyama, Niigata University Medical and Dental Hospital; Atsushi Mizokami, Kanazawa University Hospital; Tatsuya Takayama, Hamamatsu University Hospital; Takahiro Inoue, Kyoto University Hospital; Tatsuya Nakatani, Osaka City University Hospital; Kazuo Nishimura, Osaka Medical Center for Cancer and Cardiovascular Diseases; Norio Nonomura, Osaka University Hospital; Masahiro Nozawa, Kinki University Hospital; Sadanori Kamikawa, Osaka City General Hospital; Tomoharu Fukumori, Tokushima University Hospital; Shigeru Sakano, Yamaguchi University Hospital; Akito Yamaguchi, Harasanshin Hospital; Akira Yokomizo, Kyushu University Hospital; Tsukasa Igawa, Nagasaki University Hospital. We also thank the following independent data monitoring committee and independent RECIST assessment committee members: Shigeo Horie, Juntendo University Graduate School of Medicine; Shiro Hinotsu, Okayama University Hospital; Kojiro Yamamoto, Gunma University; Koichiro Akakura, Tokyo Shinjuku Medical Center; Yuichi Watanabe, National Cancer Center Hospital. Enzalutamide is being co-developed by Medivation, Inc, and Astellas Pharma, Inc. This study was funded by Astellas Pharma, Inc. The authors would like to thank Lauren Smith of Complete HealthVizion for copyediting the manuscript, which was funded by Astellas Pharma, Inc and Medivation, Inc.

\section{Compliance with ethical standards}

Conflict of interest H. A. has received personal fees from Astellas Pharma, Inc. for the submitted work and personal fees from Takeda Pharmaceutical Company Limited and Janssen Pharmaceutical K.K. outside the submitted work. Hirotsugu Uemura has received consulting fees from Astellas Pharma, Inc. for the submitted work. T. T. has received consulting fees from Astellas Pharma, Inc. S. O. has received personal fees and research funding from Astellas Pharma Inc., AstraZeneca K.K., Janssen Pharmaceutical K.K., Takeda Pharmaceutical Company Limited, and Sanofi K.K. O. O. has received consulting fees from Astellas Pharma, Inc. H. S. has received personal fees from Astellas Pharma for the submitted work; personal fees from Astellas, AstraZeneca, Takeda, and Janssen outside the submitted work; and research funds from Astellas, AstraZeneca, and Takeda outside the submitted work. M. O. has received personal fees from Astellas Pharma, Inc., Sanofi K.K., AstraZeneca K.K., ASKA Pharmaceutical, 
Janssen Pharmaceutical, Asahi Kasei Pharma Corp., Nippon Shinyaku Co., Nippon Kayaku Co., Kyorin Pharmaceutical, Medicon, Kissei Pharmaceutical, Shiongi \& Co., Pfizer Japan, Daiichi Sankyo Co., Boston Scientific Japan K.K., Taiho Pharmaceutical Co., Novartis K.K., GSK K.K., Teijin Pharma Limited, Nihon Medi-Physics Co., Otsuka Pharmaceutical Co., Japan BCG Laboratory, Takeda Pharmaceutical, Kyowa Hakko Kirin Co., Bayer Yakuhin, and Philips Electronics Japan during the conduct of the study; research funding from AstraZeneca K.K., ASKA Pharmaceutical Co., Asahi Kasei Pharma, Nippon Shinyaku Co., Ltd, Pfizer Japan, and Takeda Pharmaceutical; and encouraged donations from Astellas Pharma, Inc., Sanofi K.K., Nippon Kayaku Co., Ltd., Kyorin Pharmaceutical Col., Ltd, Medicon, Inc., Kissei Pharmaceutical Co., Ltd., Shionogi \& Co., Ltd., Daiichi Sankyo Company, Limited, Boston Scientific Japan K.K., Taiho Pharmaceutical Co., Ltd., Novartis Pharma K.K., GlaxoSmithKline K.K., Teijin Pharma Limited, Otsuka Pharmaceutical Co., Ltd, Japan BCG Laboratory, and Kyowa Hakko Kirin Co., Ltd. M. N. has received personal fees and research funding from Astellas Pharma, Inc. S. F., A. Y., and Hiroji Uemura have no conflict of interest. Y. O. has received an executive salary from Statcom, personal fees from Astellas for the submitted work; lecture fees from Daiichi Sankyo, Chugai Pharmaceutical, Shionogi, and EPS outside the submitted work; manuscript fees from Dentsu Sudler \& Hennessey and DNP Media Create outside the submitted work; and research funding from Astellas for the submitted work, and research funding from Takeda Pharmaceutical, Kyowa Hakko Kirin, and Kowa Pharmaceutical outside the submitted work. H.M. is an employee of Astellas Pharma, Inc. and owns stock from Astellas. A. S. and K.T are employees of Astellas Pharma, Inc. S. N. received personal fees from Astellas during the conduct of the study and personal fees from Takeda, AstraZeneca, Janssen and GreenPeptide outside the submitted work.

Open Access This article is distributed under the terms of the Creative Commons Attribution 4.0 International License (http://creativecommons.org/licenses/by/4.0/), which permits unrestricted use, distribution, and reproduction in any medium, provided you give appropriate credit to the original author(s) and the source, provide a link to the Creative Commons license, and indicate if changes were made.

\section{References}

1. Akaza H, Usami M, Hinotsu S et al (2004) Characteristics of patients with prostate cancer who have initially been treated by hormone therapy in Japan: J-CaP surveillance. Jpn J Clin Oncol 34:329-336

2. Cooperberg MR, Hinotsu S, Namiki M et al (2016) Trans-Pacific variation in outcomes for men treated with primary androgen deprivation therapy for prostate cancer. BJU Int 117:102-109

3. Hinotsu S, Akaza H, Usami M et al (2007) Current status of endocrine therapy for prostate cancer in Japan-analysis of primary androgen deprivation therapy on the basis of data collected by J-CaP. Jpn J Clin Oncol 37:775-781

4. Onozawa M, Hinotsu S, Tsukamoto T et al (2014) Recent trends in the initial therapy for newly diagnosed prostate cancer in Japan. Jpn J Clin Oncol 44:969-981

5. Scher HI, Halabi S, Tannock I et al (2008) Design and end points of clinical trials for patients with progressive prostate cancer and castrate levels of testosterone: recommendations of the Prostate Cancer Clinical Trials Working Group. J Clin Oncol 26:1148-1159

6. Matsumoto K, Tanaka N, Hayakawa N et al (2013) Efficacy of estramustine phosphate sodium hydrate (EMP) monotherapy in castration-resistant prostate cancer patients: report of 102 cases and review of literature. Med Oncol 30:717

7. Mottet N, Bellmunt J, Bolla M et al (2011) EAU guidelines on prostate cancer. Part II: treatment of advanced, relapsing, and castration-resistant prostate cancer. Eur Urol 59:572-583

8. Naito S, Tsukamoto T, Koga H et al (2008) Docetaxel plus prednisolone for the treatment of metastatic hormone-refractory prostate cancer: a multicenter Phase II trial in Japan. Jpn J Clin Oncol 38:365-372

9. Tannock IF, de Wit R, Berry WR et al (2004) Docetaxel plus prednisone or mitoxantrone plus prednisone for advanced prostate cancer. N Engl J Med 351:1502-1512

10. de Bono JS, Oudard S, Ozguroglu M et al (2010) Prednisone plus cabazitaxel or mitoxantrone for metastatic castration-resistant prostate cancer progressing after docetaxel treatment: a randomised open-label trial. Lancet 376:1147-1154

11. de Bono JS, Logothetis CJ, Molina A et al (2011) Abiraterone and increased survival in metastatic prostate cancer. $\mathrm{N}$ Engl $\mathrm{J}$ Med 364:1995-2005

12. Ryan CJ, Smith MR, de Bono JS et al (2013) Abiraterone in metastatic prostate cancer without previous chemotherapy. N Engl J Med 368:138-148

13. Beer TM, Armstrong AJ, Rathkopf DE et al (2014) Enzalutamide in metastatic prostate cancer before chemotherapy. N Engl J Med 371:424-433

14. Scher HI, Fizazi K, Saad F et al (2012) Increased survival with enzalutamide in prostate cancer after chemotherapy. N Engl J Med 367:1187-1197

15. Kantoff PW, Higano CS, Shore ND et al (2010) Sipuleucel-T immunotherapy for castration-resistant prostate cancer. N Engl J Med 363:411-422

16. Parker C, Nilsson S, Heinrich D et al (2013) Alpha emitter radium-223 and survival in metastatic prostate cancer. $\mathrm{N}$ Engl $\mathrm{J}$ Med 369:213-223

17. Tran C, Ouk S, Clegg NJ et al (2009) Development of a secondgeneration antiandrogen for treatment of advanced prostate cancer. Science 324:787-790

18. Bennett D, Gibbons JA, Mol R et al (2014) Validation of a method for quantifying enzalutamide and its major metabolites in human plasma by LC-MS/MS. Bioanalysis 6:737-744

19. Scher HI, Beer TM, Higano CS et al (2010) Antitumour activity of MDV3100 in castration-resistant prostate cancer: a phase 1-2 study. Lancet 375:1437-1446

20. Akaza H, Hinotsu S, Usami M et al (2009) Combined androgen blockade with bicalutamide for advanced prostate cancer: longterm follow-up of a phase 3, double-blind, randomized study for survival. Cancer 115:3437-3445

21. Venkitaraman R, Lorente D, Murthy V et al (2015) A randomised phase 2 trial of dexamethasone versus prednisolone in castrationresistant prostate cancer. Eur Urol 67:673-679

22. Kamiya N, Suzuki H, Nishimura K et al (2014) Development of nomogram to non-steroidal antiandrogen sequential alternation in prostate cancer for predictive model. Jpn J Clin Oncol 44:263-269

23. Ryan CJ, Smith MR, Fong L et al (2010) Phase I clinical trial of the CYP17 inhibitor abiraterone acetate demonstrating clinical activity in patients with castration-resistant prostate cancer who received prior ketoconazole therapy. J Clin Oncol 28:1481-1488

24. Badrising S, van der Noort V, van Oort IM et al (2014) Clinical activity and tolerability of enzalutamide (MDV3100) in patients with metastatic, castration-resistant prostate cancer who progress after docetaxel and abiraterone treatment. Cancer 120:968-975

25. Loriot Y, Bianchini D, Ileana E et al (2013) Antitumour activity of abiraterone acetate against metastatic castration-resistant 
prostate cancer progressing after docetaxel and enzalutamide (MDV3100). Ann Oncol 24:1807-1812

26. Antonarakis ES, Lu C, Wang H et al (2014) AR-V7 and resistance to enzalutamide and abiraterone in prostate cancer. $\mathrm{N}$ Engl J Med 371:1028-1038
27. Gleason DF (1992) Histologic grading of prostate cancer: a perspective. Hum Pathol 23:273-279

28. Sobin LH, Wittekind C (2002) International Union Against Cancer (UICC): TNM classification of malignant tumors, 6th edn. Wiley-Liss, New York 